\title{
Acute aortic dissection with a concomitant giant abdominal aorta aneurysm
}

\author{
Shijun Xu, MD, Lizhong Sun, MD, and Hongjia Zhang, MD
}

\footnotetext{
From the Department of Cardiac Surgery, Beijing Anzhen Hospital, Capital Medical University, Beijing, China; Beijing Institute of Heart, Lung and Blood Vessel Diseases, Beijing, China; Beijing Laboratory for Cardiovascular Precision Medicine, Beijing, China; Beijing Aortic Disease Center, Cardiovascular Surgery Center, Beijing, China; and Beijing Engineering Research Center for Vascular Prostheses, Beijing, China.

Supported by the National Key R\&D Program of China (2017YFC1308000) and the Beijing Laboratory for Cardiovascular Precision Medicine, Beijing, China (PXM2017_014226_000037).

Disclosures: Authors have nothing to disclose with regard to commercial support.

Drs Sun and Zhang contributed equally to this article.

Received for publication Dec 16, 2018; revisions received Jan 26, 2019; accepted for publication Jan 31, 2019; available ahead of print March 21, 2019.

Address for reprints: Hongjia Zhang, MD, Cardiac Surgery, Beijing Anzhen Hospital Capital Medical University, Beijing 100029, China (E-mail: zhanghongjia722@ccmu.edu.cn).

J Thorac Cardiovasc Surg 2019;158:e161-2

$0022-5223 / \$ 36.00$

Copyright () 2019 by The American Association for Thoracic Surgery https://doi.org/10.1016/j.jtcvs.2019.01.123
}

- Video clip is available online.

A 28-year-old man presented to the emergency department with severe chest and back pain. He had a history of a thoracic endovascular aortic repair procedure 4 years

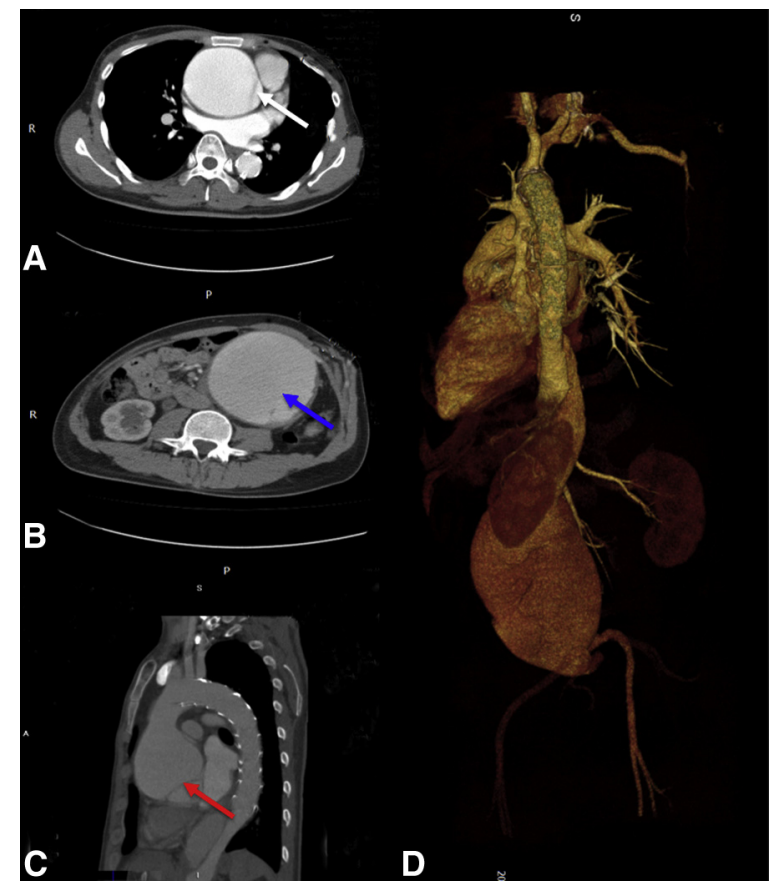

FIGURE 1. A through $\mathrm{C}$, Computed tomographic angiography of the thorax and abdomen revealed a type I DeBakey aortic dissection (A; white arrow) and an abdominal aortic aneurysm (B; blue arrows). The dissection involved the ascending aorta $(\mathrm{C}$; red arrow). $\mathrm{D}$, Three-dimensional reconstruction of the overall aorta.

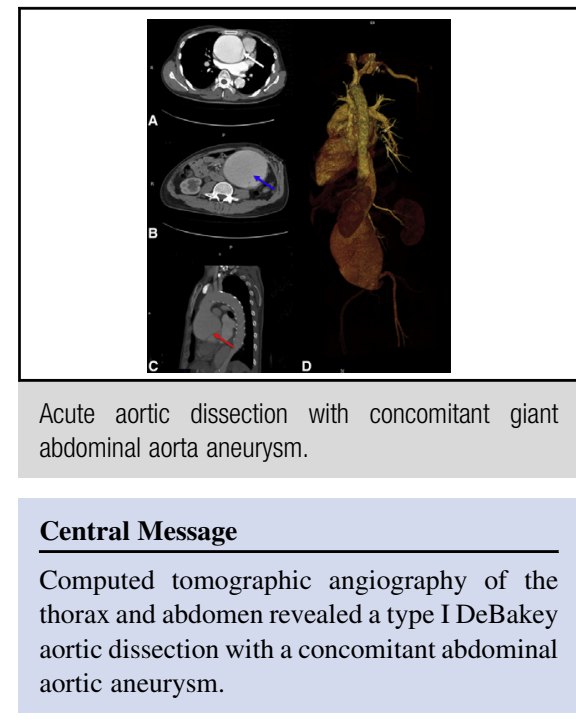

See Commentary on page e163.

previously for a type III DeBakey aortic dissection. After that procedure, he was not followed up closely. The patient had neither clinical manifestations of Marfan syndrome nor a family history of hereditary aortic disease. He had no history of hypertension, and he did not receive any medication after the thoracic endovascular aortic repair procedure. His troponin I level was normal, he reported no shortness of breath and fatigue, and electrocardiography did not show evidence of ischemic changes. Computed tomographic angiography of the thorax and abdomen revealed a type I DeBakey aortic dissection (Figure 1, A; white arrow) and an abdominal aortic aneurysm (Figure 1, $B ;$ blue arrows). The dissection involved the ascending aorta (Figure 1, C; red arrow). Computed tomographic angiography of the thorax and abdomen also revealed dilation of the ascending aorta $(75.5 \mathrm{~mm} \times 160.6 \mathrm{~mm})$ and the abdominal aorta $(127.3 \mathrm{~mm} \times 194.2 \mathrm{~mm})$. Because of the absence of associated symptoms, the abdominal aneurysm was considered to be chronic. The patient underwent a Bentall procedure and abdominal aortic replacement at the same time. Examination of the aortic pathologic specimen showed that the elastic fibers in the middle of the aortic wall were thinner, disordered, and fibrotic, and the smooth muscle cells were disordered. The patient recovered smoothly after the operation. Three months later, the patient's condition was better, and he received $\beta$-blocker therapy and routine follow-up care (Video 1). 


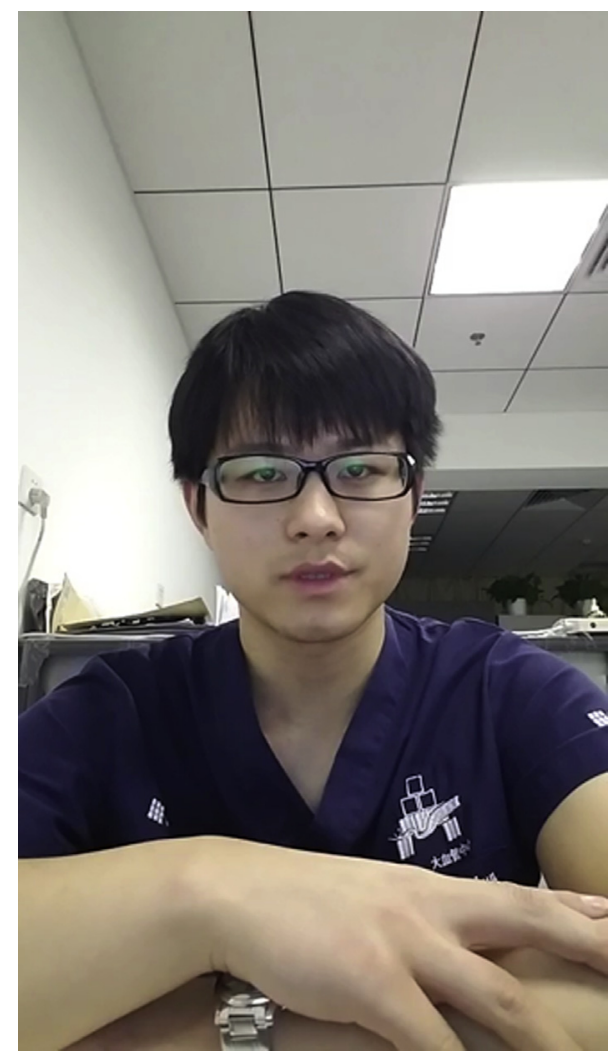

VIDEO 1. This case was completely different from previously reported abdominal aortic aneurysm because of its large size and concomitant ascending dissection. The Bentall procedure and abdominal aortic replacement were performed at the same time. Video available at: https://www.jtcvs.org/article/ S0022-5223(19)30358-7/fulltext. 